\title{
Cross-cultural adaptation and psychometric assessment of the Portuguese language version of the Eating and Appraisal due to Emotions and Stress (EADES) Questionnaire in Brazilian adults
}

\author{
Priscila Carvalho Santos ${ }^{1}$ (1) - Wanderson Roberto da Silva ${ }^{1}$ (1) . João Marôco ${ }^{2}$ (1) . \\ Juliana Alvares Duarte Bonini Campos ${ }^{1} \mathbb{0}$
}

Received: 1 May 2021 / Accepted: 18 September 2021

(c) The Author(s), under exclusive licence to Springer Nature Switzerland AG 2021

\begin{abstract}
Purposes To develop a Brazilian Portuguese version of the Eating and Appraisal due to Emotions and Stress (EADES) Questionnaire and estimate the psychometric properties of the EADES factorial model for young Brazilian adults and also to assess the association between EADES factors and age, body mass index (BMI), and economic level.

Methods The cross-cultural adaptation was performed using a standardized protocol. The psychometric properties were assessed separately for each sex. A structural model for each sex was developed to investigate the influence of age, economic level, and BMI on the EADES factors.

Results A total of 1240 participants completed the study [65.8\% female, mean age 23.91 ( $\mathrm{SD}=5.03$ ) years]. The EADES original factorial model did not present good psychometric properties. Then, a factorial model proposed for a Mexican sample was tested and a different model was fitted for each sex. The results showed that younger women have lower self-efficacy and self-confidence and poorer assessment of resources and coping skills. Women with a higher economic level have lower self-efficacy. Higher BMI was associated with lower self-efficacy and self-confidence in both sexes. Younger men have lower self-efficacy and poorer assessment of resources and coping skills.

Conclusions The Brazilian Portuguese version of the EADES provided valid and reliable data after refinement, and a different model was fitted for each sex. Sex, age, BMI, and economic level were significantly associated with the EADES factors. Level of evidence Level V, descriptive cross-sectional study.
\end{abstract}

Keywords Cross-cultural adaptation $\cdot$ Eating $\cdot$ Stress $\cdot$ Emotion $\cdot$ Psychometrics $\cdot$ Scale

\section{Introduction}

Emotional eating can be understood as a behavior to select and eat specific foods in an attempt to change, relieve, or reinforce feelings, as well as dealing with positive or negative experiences [1,2]. Positive emotions such as happiness can influence food intake to reinforce the positive feeling,

Juliana Alvares Duarte Bonini Campos

juliana.campos@unesp.br

1 Department of Biological Sciences, School of Pharmaceutical Sciences, São Paulo State University (UNESP, Campus Araraquara), Rodovia Araraquara-Jaú, km 01, Araraquara, São Paulo Zip Code: 14800-903, Brazil

2 William James Center for Research (WJCR), ISPAUniversity Institute of Psychological, Social and Life Sciences, Lisbon, Portugal especially in socialization activities [3]. On the other hand, negative emotions and stress - the coordinated response of the body to any type of change that causes physical, emotional, or psychological strain [4]—also can influence food intake, but as a way to reduce the psychological discomfort [2] and serving as a coping strategy [5]. Konttinen et al. [6] emphasize that negative emotions can alter the hunger and satiety cues and affect the eating behavior of individuals, such as motivation to eat, food choice and quantity, and eating speed. In this way, using food to control or relieve discomfort [7] can increase or decrease the quantity of eaten food $[1,8]$ and trigger risky eating behaviors, such as restrictive eating, binge eating, and disordered eating [1, 9-11]. These changes can impact people's physical $[2,5]$ and mental [11] health and could result in the development of eating disorders $[8,12,13]$. Thus, exploring how individuals use food to cope with emotions and stress is an important step 
for the development of efficient health protocols and diet interventions.

As emotional eating cannot be directly measured (because it is a latent variable), the use of specific tools is essential. For that, the literature has suggested the use of psychometric instruments [7, 13-18] to assess eating based on emotion or stress as a unique concept or as part of an eating behavior. Among these instruments, the Eating and Appraisal due to Emotions and Stress (EADES) Questionnaire stands out for considering not only the effects of specific emotions and stress on eating behavior but also eating as a coping and adaptive strategy. Differently, the Positive-Negative Emotional Eating Scale [16] and the Salzburg Emotional Eating Scale [13] do not assess copying mechanisms.

The EADES was developed based on the Transactional Model of Stress and Coping (TMSC) considering the process of dealing with emotions and stress and different degrees and types of reaction of individuals. Each person tends to react differently to situations and life events and how one interprets and reacts to them can lead to an effective or ineffective coping response. Thus, the theoretical concept behind the EADES can help to understand these differences that involve the triggering event, its cognitive evaluation, and the decision on how to cope with the event [7].

The EADES was originally proposed by Ozier et al. [7] in the English language and tested in an American sample. The questionnaire was then translated by Lazarevich et al. [2] to the Spanish language and tested in a Mexican sample. Both studies [2,7] evaluated the psychometric properties of the EADES and fitted different structural models to the samples. Currently, no Portuguese version of this instrument is available and there is no validity and reliability study of the EADES in a Brazilian context. Considering that studies carried out in Brazil reported high rates of disordered and emotional eating [19] and a positive association between unhealthy eating habits and psychological symptoms, such as depression, anxiety, [20] and stress [21], a Brazilian version of the EADES can be useful to track the signs and symptoms of emotional eating. Additionally, this instrument can help to identify groups at high risk for emotional eating that need a special attention of professionals.

Emotions and stressful events can have different effects in eating habits of women and men, with women being more affected by emotional eating [22,23]. A review carried out by Jiang et al. (2014) suggests that food seems to be used more often by women as a way of coping with, and feeling better towards stress [24]. The eating behavior can also be influenced by the body mass index (BMI), as people with a higher BMI have higher perceived stress [13] and are more susceptible to emotional eating [13, 23]. Geliebter et al. [15] reported that overweight individuals eat more during negative emotional states than people with normal weight or underweight. In contrast, during positive emotional states, underweight participants eat more than those with normal weight or overweight. Age has also been linked to emotional eating, with older individuals appearing to be less affected $[25,26]$. Finally, the economic level may also influence the eating behavior of individuals [27], but such relationship is still not well established and further investigations are needed.

Therefore, the purposes of this study were to carry out the cross-cultural adaptation of EADES to the Portuguese language, assess the validity and reliability of the data obtained with EADES in young Brazilian adults, and verify the association between individual characteristics and concepts evaluated by the EADES. Our hypotheses were that the Portuguese version of the EADES will have good understandability and different factorial models will be fitted for female and male samples. We also hypothesized and that age, BMI, and economic level will be associated with the concepts evaluated by EADES.

\section{Methods}

\section{Study design and sample size}

This was an observational, cross-sectional study with a non-probabilistic sampling method. The calculation of the minimum sample size was based on the need for at least five respondents for each item of the instrument [28]. As the EADES was originally proposed with 49 items, the minimum estimated sample size was 245 participants. A rate of $10 \%$ was added to compensate for possible losses, which resulted in a sample of 273 individuals for each sex, as the analyses were performed separately for female and male.

\section{Participants}

People aged between 18 and 35 years from Brazil were invited to participate in the study. Exclusion criteria were pregnant or lactating women, and blindness. Participants provided information of their sex (female or male), age (years), body weight $(\mathrm{kg})$, height $(\mathrm{m})$, and economic level. Weight and height were used to calculate BMI for the anthropometric nutritional status [29, 30]. To estimate the economic level, the Brazil Economic Classification Criteria [31] was used, which is based on the family's estimated gross average monthly income $(\mathrm{R} \$ 25,554.33=$ high economy class [A]; R \$ 8,460.39=medium-high economic class [B]; R\$ 2,417.04= medium-low economic class [C]; $\mathrm{R} \$ 719.81=$ low economic class [D and E]; the exchange rate as of July/2021 was $1 \mathrm{USD}=5.17 \mathrm{BRL}$. In total, 1,362 people agreed to participate in the study, of which 1,240 completed all EADES items and were included in the analysis $($ loss rate $=8.9 \%)$. 


\section{Measuring instrument}

The EADES was initially developed with 54 items and after refinement, a set of 49 items was presented [7]. Twenty-one items are formulated with an inverted response scale in relation to the others (inverted items: 4, 7, 10, 12, 13, 14, 15, $20,23,28,31,35,36,39,41,43,45,46,50,52$, and 54). The response options are given as a five-point Likert scale ( $1=$ strongly disagree, $2=$ disagree, $3=$ neither disagree nor agree, $4=$ agree and $5=$ strongly agree). The authors proposed a three-factor model including "Emotion- and StressRelated Eating" (items: 3, 4, 10, 13, 14, 16, 21, 23, 26, 28, $30,34,35,36,38,39,41,43,45,47,49,50,52$, and 54), "Appraisal of Ability and Resources to Cope with Emotions and Stress" (items 2, 5, 6, 7, 8, 11, 18, 20, 22, 24, 25, 27, 29, 32, 33, 37, 40, 46, 51 and 53) and "Appraisal of Outside Stressors/Influences" (items: 12, 15, 31, 42, and 48). This structure (3 factors and 49 items) was found using Exploratory Factor Analysis (EFA) and presented acceptable internal consistency (Cronbach's alpha $[\alpha]$ : all items $=0.95$ and factors $=0.65-0.95$ ). In the original study, data on construct validity were not presented.

Afterwards, Lazarevich et al. [2] cross-culturally adapted the three-factor original EADES to the Spanish language using a sample of university students in Mexico. The authors found that the factor "Appraisal of Outside Stressors/Influences" had low internal consistency $(\alpha=0.54)$. Thus, a new factorial model for the EADES was explored and proposed for the Mexican context. This was composed of 40 items (all with factor loadings $>0.40$ ) distributed in three factors as follows: "Self-efficacy in Emotion- and Stress-Related Eating" (items: 4, 13, 14, 23, 28, 35, 36, 39, 43, 45, 50, and 54), "Self-confidence in Emotion- and Stress-Related Eating" (items: 3, 16, 21, 26, 30, 34, 38, 47, and 49), and "Appraisal of Resources and Ability to Cope" (items: 2, 5, $6,7,8,11,18,20,22,24,25,27,29,32,33,37,40,51$, and 53). The first factor of the original model (Emotionand Stress-Related Eating) was subdivided into two factors ("Self-efficacy in Emotion- and Stress-Related Eating" and "Self -confidence in Emotion- and Stress-Related Eating") in the Mexican model. The second factor of the original model ("Appraisal of Ability and Resources to Cope with Emotions and Stress") was relocated to the third factor in the Mexican model and renamed "Appraisal of Resources and Ability to Cope". Finally, the third factor of the original instrument ("Appraisal of Outside Stressors/Influences") was excluded, since the items had low factor loading. This structure presented adequate internal consistency $(\alpha=0.84-0.91)$ and test-retest reliability (intraclass correlation coefficient $=0.88, p<0.01$ ) for the Mexican data; however, the construct validity was not evaluated.

In the present study, the original model (i.e., 3 factors and 49 items) and Mexican model (i.e., 3 factors and 40 items) of the EADES were tested and evaluated in the Brazilian sample (see Supplementary Material-Fig. 1).

\section{Cross-cultural adaptation}

The cross-cultural adaptation of the EADES to the Brazilian Portuguese language was carried out, and the semantic, idiomatic, conceptual, and cultural equivalences were analyzed [32]. First, the English version of the EADES was sent to four independent translators with Portuguese as first language and knowledge of the American culture to translate the content to Portuguese, following the 2009 orthographic agreement of Portuguese-speaking countries. Afterwards, the translated versions were compared by the authors of this study and a single initial Portuguese language version was established and sent to an independent native English translator with knowledge of Portuguese and not familiar with the original EADES for back translation into English. The content of both versions was compared and equivalence was found. In addition, the Portuguese version was analyzed by two Psychometrics and Health specialists who considered the version produced to be adequate for the Brazilian context. This version was tested in a pilot study with a sample of 32 individuals $(71.9 \%$ female, mean age 28.9 [SD=6.6] years) to check the understandability of the items. As there were no reports of difficulty in filling out or understanding the questionnaire, the Portuguese version was considered adequate (see Supplementary Material-Appendix 1).

\section{Procedures and ethical aspects}

First, students, technical-administrative staff, and professors from a public university in Brazil were invited to participate by e-mails, social networks, and personal invitations. Those who agreed to participate filled out the EADES Portuguese version and a sociodemographic questionnaire using a paperand-pencil format. Then, snowball sampling was adopted, where participants indicated new individuals to participate in the study. This study was approved by the Human Research Ethics Committee of the School of Pharmaceutical Sciences of UNESP (C.A.A.E.: 11,469,719.1.0000.5426) and all participants signed a consent form.

\section{Data analysis}

The psychometric properties of both the original and Mexican models $[2,7]$ were evaluated initially for the total sample and then separately for the female and male samples. The descriptive statistical analysis (i.e., mean, median, mode, standard deviation, skewness, and kurtosis) of the responses given to the EADES items was performed to verify data distribution. Absolute values of skewness and kurtosis greater 
than 3 and 7, respectively, indicated non-normal data distribution [33].

Construct validity was assessed based on factorial, convergent, and discriminant validities. The factorial validity was estimated using the confirmatory factor analysis (CFA) through the weighted least squares mean and variance adjusted (WLSMV) estimator. To assess the fit of the models to the data, the chi-square for degrees of freedom ratio $\left(\chi^{2} / d f\right)$, Comparative Fit Index (CFI), Tucker-Lewis Index (TLI), and Root Mean Square Error of Approximation (RMSEA) with a $90 \%$ confidence interval (CI) were calculated. The fit was considered acceptable when $\chi^{2} / d f \leq 5.0$, CFI and TLI $\geq 0.90$, and RMSEA $\leq 0.10$ [33]. The factor loading $(\lambda)$ of each item was also estimated and items with values less than 0.40 were eliminated. In case of poor fit of the model, the modification indices (MI), calculated using the Lagrange Multiplier method, greater than 11 were inspected [33].

Convergent validity was analyzed with the average variance extracted (AVE) and values $\geq 0.50$ were considered adequate [34]. The discriminant validity was estimated using correlational analysis and AVE. $\mathrm{AVE}_{\mathrm{i}}$ and $\mathrm{AVE}_{\mathrm{j}}$ values for two correlated factors greater than the square of the correlation between factors $\left(r_{\mathrm{ij}}^{2}\right)$ indicated there was adequate discriminant validity [33, 34].

To assess the reliability of the EADES factors, the Composite Reliability (CR) and the ordinal alpha coefficient $(\alpha)$ were estimated. Values of $\mathrm{CR}$ and $\alpha \geq 0.70$ were considered adequate $[33,34]$. The program $\mathrm{R}(\mathrm{R}$ Core Team, 2019) and the packages "lavaan" [35] and "semTools" [36] were used for this analysis.

\section{Structural model}

A structural model was built to investigate the influence of age, BMI, and economic level (independent variables) on EADES factors (dependent variables). Considering that the EADES fitted model was different for female and male samples, a structural model for each sex was developed and tested. For interpreting the EADES factors in the structural models, it was considered that for the "Self-efficacy in Emotion- and Stress-Related Eating" factor, the higher the scores, the less the self-efficacy. In this way, items 7 and 20 had their answers inverted to be in the same direction as the others. For the other factors ("Self-confidence in Emotionand Stress-Related Eating" and "Appraisal of Resources and Ability to Cope"), the lower the score, the lower the selfconfidence and the less the resources and coping skills.

To verify the fit of the structural models to the data, the indices $\chi^{2} / d f$, CFI, TLI, and RMSEA were used according to the values mentioned above. The hypothetically causal trajectories $(\beta)$ were estimated and evaluated using the $\mathrm{z}$ test. A significance level of 5\% was adopted for decision making
[33]. This assessment was carried out in the MPLUS program v.7.2 (Muthén \& Muthén, Los Angeles, CA).

\section{Results}

The mean age of the participants was $23.9(S D=5.0)$ years, and $65.8 \%$ was female. For females, the average age was $23.5(S D=5.0)$ years and for males, $24.6(S D=5.1)$ years. The average BMI for the total sample was $24.3(S D=4.4)$ $\mathrm{kg} / \mathrm{m}^{2}$. For female, the average BMI was $23.6(S D=4.3)$ $\mathrm{kg} / \mathrm{m}^{2}$ and for male, $25.5(S D=4.3) \mathrm{kg} / \mathrm{m}^{2}$. Regarding the anthropometric nutritional status, $2.6 \%$ of participants were classified as underweight (female $=3.0 \%$, male $=1.7 \%$ ), $60 \%$ as normal weight $($ female $=63.3 \%$, male $=48.1 \%$ ), $28.1 \%$ as overweight (female $=23.2 \%$, male $=37.2 \%$ ), and $9.4 \%$ as obese (female $=7.4 \%$, male $=13 \%$ ). As for the economic level, $29.1 \%$ were classified as high (class A: female $=27.4 \%$, male $=32.5 \%$ ), $57.3 \%$, medium - high (class B: female $=60.2 \%$, male $=51.8 \%$ ), $13.2 \%$, medium-low (class C: female $=11.9 \%$, male $=15.5 \%$ ), and $4.0 \%$, low (classes D and E: female $=5 \%$, male $=2 \%$ ).

The descriptive statistics of the EADES responses is shown in Table 1. None of the items presented discrepant values of skewness and kurtosis. Table 2 shows the factorial loading of the EADES items for total, female, and male samples considering the original model and the Mexican model.

For the original model, low factor loading was observed in items $2,10,18,29,41$, and 52 in the total sample, items $2,10,18,24,29,41$, and 52 in the female sample, and 2, 10, $15,18,29,42$, and 52 in the male sample. For the Mexican model, low factor loading was observed in items 2,18 , and 29 in the total sample, 2, 18, 24, and 29 in the female sample, and 2, 18, and 29 in the male sample.

Table 3 shows the psychometric properties for each tested model. The original model did not show good fit to any of the samples (total, female, and male). In an attempt to improve the fit of this model, items with factor loading $<0.40$ were excluded and strong correlations between item errors (MI > 240.34) were inserted. However, even after these modifications, the original model did not fit any of the samples, nor did it present adequate convergent validity. On the other hand, the reliability of the original model was adequate (see Table 3), except for the "Appraisal of Outside Stressors/Influences" factor in the male sample, which was borderline $(\alpha=0.67, \mathrm{CR}=0.66)$. An EFA was carried out to verify the distribution of factors for the Brazilian data (data not shown). In this analysis, we found a set of ten factors without a well-established background. Afterwards, we established a three-factor structure in the EFA and found a model similar to the Mexican model. Therefore, was decided to test the Mexican model in our data. 
Table 1 Descriptive statistics of the Eating and Appraisal due to Emotions and Stress (EADES) Questionnaire for sample of Brazilian adults (Total sample: $n=1240$; Female sample: $n=815$; Male sample: $n=425$ )

\begin{tabular}{|c|c|c|c|c|c|c|c|c|c|c|c|c|c|c|c|c|c|c|}
\hline \multirow[b]{2}{*}{ Item } & \multicolumn{6}{|c|}{ Total sample } & \multicolumn{6}{|c|}{ Female sample } & \multicolumn{6}{|c|}{ Male sample } \\
\hline & M & $\mathrm{Md}$ & Mo & SD & $\mathrm{Sk}$ & $\mathrm{Ku}$ & M & $\mathrm{Md}$ & Mo & SD & Sk & $\mathrm{Ku}$ & M & $\mathrm{Md}$ & Mo & SD & Sk & $\mathrm{Ku}$ \\
\hline 2 & 3.99 & 4 & 4 & 0.89 & -1.03 & 1.33 & 3.99 & 4 & 4 & 0.88 & -0.97 & 1.11 & 4.00 & 4 & 4 & 0.90 & -1.15 & 1.73 \\
\hline 3 & 3.53 & 4 & 4 & 0.95 & -0.73 & 0.17 & 3.49 & 4 & 4 & 0.95 & -0.80 & 0.20 & 3.61 & 4 & 4 & 0.93 & -0.60 & 0.03 \\
\hline 4 & 2.89 & 3 & 2 & 1.25 & 0.12 & -1.15 & 3.08 & 3 & 4 & 1.25 & -0.06 & -1.16 & 2.54 & 2 & 2 & 1.19 & 0.48 & -0.79 \\
\hline 5 & 3.56 & 4 & 4 & 0.89 & -0.76 & 0.53 & 3.49 & 4 & 4 & 0.90 & -0.74 & 0.42 & 3.68 & 4 & 4 & 0.86 & -0.79 & 0.78 \\
\hline 6 & 3.56 & 4 & 4 & 0.85 & -0.80 & 0.55 & 3.48 & 4 & 4 & 0.86 & -0.78 & 0.39 & 3.71 & 4 & 4 & 0.82 & -0.85 & 0.94 \\
\hline 7 & 2.50 & 2 & 2 & 1.05 & 0.46 & -0.45 & 2.58 & 2 & 2 & 1.07 & 0.38 & -0.54 & 2.33 & 2 & 2 & 0.99 & 0.61 & -0.19 \\
\hline 8 & 3.78 & 4 & 4 & 0.73 & -0.93 & 1.70 & 3.78 & 4 & 4 & 0.72 & -0.84 & 1.42 & 3.79 & 4 & 4 & 0.77 & -1.05 & 2.10 \\
\hline 10 & 2.73 & 3 & 2 & 1.05 & 0.22 & -0.77 & 2.74 & 3 & 2 & 1.06 & 0.24 & -0.78 & 2.71 & 3 & 2 & 1.04 & 0.16 & -0.76 \\
\hline 11 & 3.76 & 4 & 4 & 0.87 & -0.82 & 0.78 & 3.73 & 4 & 4 & 0.87 & -0.82 & 0.68 & 3.81 & 4 & 4 & 0.85 & -0.82 & 1.01 \\
\hline 12 & 3.33 & 4 & 4 & 1.15 & -0.41 & -0.70 & 3.38 & 4 & 4 & 1.14 & -0.49 & -0.58 & 3.22 & 3 & 4 & 1.15 & -0.26 & -0.87 \\
\hline 13 & 3.24 & 3 & 4 & 1.19 & -0.28 & -0.94 & 3.41 & 4 & 4 & 1.17 & -0.43 & -0.78 & 2.92 & 3 & 4 & 1.17 & -0.04 & -1.02 \\
\hline 14 & 2.77 & 3 & 2 & 1.22 & 0.17 & -1.08 & 3.03 & 3 & 4 & 1.19 & -0.03 & -1.09 & 2.28 & 2 & 2 & 1.10 & 0.58 & -0.57 \\
\hline 15 & 3.51 & 4 & 4 & 1.01 & -0.54 & -0.14 & 3.53 & 4 & 4 & 1.00 & -0.56 & -0.03 & 3.47 & 4 & 4 & 1.03 & -0.49 & -0.30 \\
\hline 16 & 3.17 & 3 & 4 & 1.09 & -0.26 & -0.81 & 3.13 & 3 & 4 & 1.09 & -0.23 & -0.85 & 3.26 & 3 & 4 & 1.09 & -0.31 & -0.70 \\
\hline 18 & 3.79 & 4 & 4 & 0.84 & -0.87 & 1.24 & 3.85 & 4 & 4 & 0.81 & -0.90 & 1.58 & 3.67 & 4 & 4 & 0.90 & -0.80 & 0.72 \\
\hline 20 & 2.63 & 2 & 2 & 1.09 & 0.39 & -0.71 & 2.78 & 3 & 2 & 1.09 & 0.29 & -0.86 & 2.34 & 2 & 2 & 1.01 & 0.58 & -0.26 \\
\hline 21 & 3.19 & 3 & 4 & 1.09 & -0.27 & -0.81 & 3.08 & 3 & 4 & 1.08 & -0.18 & -0.91 & 3.39 & 4 & 4 & 1.07 & -0.46 & -0.48 \\
\hline 22 & 3.28 & 3 & 4 & 0.97 & -0.37 & -0.31 & 3.22 & 3 & 4 & 0.97 & -0.40 & -0.33 & 3.39 & 3 & 4 & 0.97 & -0.33 & -0.32 \\
\hline 23 & 2.14 & 2 & 2 & 1.02 & 0.89 & 0.25 & 2.14 & 2 & 2 & 1.03 & 0.93 & 0.37 & 2.13 & 2 & 2 & 0.99 & 0.78 & -0.02 \\
\hline 24 & 3.46 & 4 & 4 & 1.04 & -0.60 & -0.29 & 3.40 & 4 & 4 & 1.05 & -0.57 & -0.36 & 3.57 & 4 & 4 & 1.00 & -0.67 & -0.14 \\
\hline 25 & 3.71 & 4 & 4 & 0.96 & -0.91 & 0.55 & 3.65 & 4 & 4 & 0.97 & -0.81 & 0.22 & 3.84 & 4 & 4 & 0.93 & -1.14 & 1.45 \\
\hline 26 & 2.99 & 3 & 4 & 1.13 & -0.05 & -1.01 & 2.85 & 3 & 2 & 1.10 & 0.06 & -0.99 & 3.26 & 4 & 4 & 1.14 & -0.31 & -0.87 \\
\hline 27 & 3.11 & 3 & 3 & 0.99 & -0.30 & -0.51 & 2.95 & 3 & 3 & 0.99 & -0.18 & -0.60 & 3.41 & 4 & 4 & 0.92 & -0.53 & -0.03 \\
\hline 28 & 2.18 & 2 & 2 & 1.05 & 0.81 & 0.02 & 2.28 & 2 & 2 & 1.09 & 0.71 & -0.21 & 1.98 & 2 & 2 & 0.95 & 0.97 & 0.57 \\
\hline 29 & 3.15 & 3 & 4 & 1.16 & -0.28 & -0.86 & 3.24 & 3 & 4 & 1.14 & -0.35 & -0.75 & 2.96 & 3 & 4 & 1.18 & -0.14 & -1.00 \\
\hline 30 & 3.02 & 3 & 4 & 1.11 & -0.11 & -0.85 & 2.89 & 3 & 3 & 1.07 & 0.00 & -0.81 & 3.25 & 3 & 4 & 1.14 & -0.38 & -0.72 \\
\hline 31 & 2.93 & 3 & 3 & 1.08 & -0.13 & -0.80 & 2.99 & 3 & 3 & 1.08 & -0.16 & -0.76 & 2.81 & 3 & 3 & 1.05 & -0.07 & -0.85 \\
\hline 32 & 3.34 & 4 & 4 & 0.99 & -0.47 & -0.40 & 3.33 & 4 & 4 & 0.97 & -0.48 & -0.38 & 3.35 & 4 & 4 & 1.03 & -0.46 & -0.44 \\
\hline 33 & 3.80 & 4 & 4 & 0.86 & -1.01 & 1.27 & 3.79 & 4 & 4 & 0.85 & -1.01 & 1.31 & 3.82 & 4 & 4 & 0.88 & -1.02 & 1.23 \\
\hline 34 & 3.06 & 3 & 4 & 1.08 & -0.09 & -0.86 & 2.90 & 3 & 2 & 1.04 & 0.01 & -0.83 & 3.37 & 4 & 4 & 1.08 & -0.37 & -0.71 \\
\hline 35 & 2.37 & 2 & 2 & 1.12 & 0.57 & -0.47 & 4.46 & 2 & 2 & 1.15 & 0.49 & -0.62 & 2.20 & 2 & 2 & 1.04 & 0.69 & -0.12 \\
\hline 36 & 3.84 & 3 & 2 & 1.19 & 0.13 & -1.07 & 3.09 & 3 & 4 & 1.17 & -0.10 & -1.06 & 2.36 & 2 & 2 & 1.05 & 0.53 & -0.55 \\
\hline 37 & 3.41 & 4 & 4 & 0.95 & -0.68 & -0.03 & 3.27 & 3 & 4 & 0.97 & -0.58 & -0.31 & 3.69 & 4 & 4 & 0.86 & -0.86 & 0.86 \\
\hline 38 & 3.00 & 3 & 4 & 1.08 & -0.05 & -0.87 & 2.88 & 3 & 2 & 1.04 & 0.05 & -0.83 & 3.24 & 3 & 4 & 1.10 & -0.31 & -0.77 \\
\hline 39 & 3.02 & 3 & 4 & 1.25 & -0.11 & -1.09 & 3.35 & 4 & 4 & 1.16 & -0.37 & -0.75 & 2.38 & 2 & 2 & 1.17 & 0.43 & -0.93 \\
\hline 40 & 3.43 & 4 & 4 & 0.90 & -0.49 & 0.13 & 3.42 & 4 & 4 & 0.88 & -0.45 & 0.04 & 3.44 & 4 & 4 & 0.95 & -0.54 & 0.22 \\
\hline 41 & 2.60 & 2 & 2 & 1.07 & 0.28 & -0.85 & 2.59 & 2 & 2 & 1.02 & 0.34 & -0.74 & 2.62 & 2 & 2 & 1.14 & 0.19 & -1.04 \\
\hline 42 & 3.03 & 3 & 3 & 0.94 & 0.14 & -0.29 & 2.98 & 3 & 3 & 0.94 & 0.23 & -0.26 & 3.10 & 3 & 3 & 0.94 & -0.04 & -0.26 \\
\hline 43 & 2.61 & 2 & 2 & 1.12 & 0.36 & -0.78 & 2.72 & 3 & 2 & 1.12 & 0.27 & -0.87 & 2.40 & 2 & 2 & 1.08 & 0.53 & -0.51 \\
\hline 45 & 2.84 & 3 & 2 & 1.24 & 0.12 & -1.19 & 3.07 & 3 & 4 & 1.23 & -0.09 & -1.20 & 2.40 & 2 & 2 & 1.14 & 0.52 & -0.72 \\
\hline 46 & 2.63 & 2 & 2 & 0.97 & 0.38 & -0.52 & 2.70 & 3 & 2 & 0.97 & 0.33 & -0.62 & 2.47 & 2 & 2 & 0.97 & 0.50 & -0.24 \\
\hline 47 & 2.69 & 2 & 2 & 1.17 & 0.26 & -1.00 & 2.51 & 2 & 2 & 1.14 & 0.42 & -0.85 & 3.03 & 3 & 4 & 1.14 & -0.03 & -1.01 \\
\hline 48 & 2.77 & 3 & 3 & 1.00 & 0.12 & -0.62 & 2.62 & 3 & 2 & 0.98 & 0.21 & -0.54 & 3.05 & 3 & 3 & 0.99 & -0.08 & -0.57 \\
\hline 49 & 3.63 & 4 & 4 & 0.96 & -0.76 & 0.23 & 3.59 & 4 & 4 & 0.96 & -0.83 & 0.33 & 3.69 & 4 & 4 & 0.96 & -0.65 & 0.03 \\
\hline 50 & 3.40 & 4 & 4 & 1.34 & -0.49 & -1.03 & 3.68 & 4 & 4 & 1.27 & -0.81 & -0.49 & 2.87 & 3 & 4 & 1.33 & 0.02 & -1.25 \\
\hline 51 & 3.42 & 4 & 4 & 0.93 & -0.64 & 0.22 & 3.36 & 3 & 4 & 0.93 & -0.62 & 0.16 & 3.53 & 4 & 4 & 0.93 & -0.71 & 0.42 \\
\hline 52 & 3.11 & 3 & 4 & 0.95 & -0.45 & -0.42 & 3.14 & 3 & 4 & 0.91 & -0.44 & -0.39 & 3.05 & 3 & 4 & 1.03 & -0.42 & -0.56 \\
\hline
\end{tabular}


Table 1 (continued)

\begin{tabular}{|c|c|c|c|c|c|c|c|c|c|c|c|c|c|c|c|c|c|c|}
\hline \multirow[b]{2}{*}{ Item } & \multicolumn{6}{|c|}{ Total sample } & \multicolumn{6}{|c|}{ Female sample } & \multicolumn{6}{|c|}{ Male sample } \\
\hline & $\mathrm{M}$ & $\mathrm{Md}$ & Mo & SD & Sk & $\mathrm{Ku}$ & $\mathrm{M}$ & $\mathrm{Md}$ & Mo & $\mathrm{SD}$ & Sk & $\mathrm{Ku}$ & $\mathrm{M}$ & $\mathrm{Md}$ & Mo & $\mathrm{SD}$ & $\mathrm{Sk}$ & $\mathrm{Ku}$ \\
\hline 53 & 3.96 & 4 & 4 & 0.66 & -0.85 & 2.15 & 3.94 & 4 & 4 & 0.67 & -0.77 & 1.74 & 3.99 & 4 & 4 & 0.65 & -1.02 & 3.00 \\
\hline 54 & 2.16 & 2 & 2 & 1.02 & 0.80 & 0.05 & 2.20 & 2 & 2 & 1.05 & 0.81 & 0.04 & 2.08 & 2 & 2 & 0.97 & 0.75 & -0.07 \\
\hline
\end{tabular}

Note. $M$ mean, $M d$ median, $M o$ mode, $S D$ standard deviation, $S k$ skewness, $K u$ kurtosis

For the Mexican factorial model, an acceptable fit was observed for all samples (see Table 3). However, some items presented low factor loading (total sample: items 2, 18, and 29; female sample: items 2, 18, 24, and 29; male sample: items 2, 18, and 29) and were excluded. This contributed to an adequate convergent validity for the factors "Self-efficacy in Emotion- and Stress-Related Eating" and "Self-confidence in Emotion- and Stress-Related Eating" (see Table 3). On the other hand, the AVE values were not adequate $(<0.50)$ for the "Appraisal of Resources and Ability to Cope" factor in any of the samples. For the discriminant validity and reliability, the fitted models presented good indicators (see Table 3). As a different model was fitted for each sex-indicating that the EADES concepts are operationalized differently in females and males-the use of the factorial model for the total sample is not recommended. From this perspective, the fitted female model was composed of 3 factors and 36 items and the fitted male model was composed of 3 factors and 37 items (see Supplementary Material-Fig. 2).

Table 4 shows the results of the structural models built based on the EADES factorial models fitted for each sex. Both female and male models showed non-significant paths $(p>0.05)$ and were eliminated, with only significant relationships remaining in the final models. For the female model, age was significantly associated to all factors of the EADES, the economic level was associated with the "Selfefficacy in Emotion- and Stress-Related Eating" factor, and BMI, with the factors "Self-efficacy in Emotion- and Stress-Related Eating" and "Self-confidence in Emotionand Stress-Related Eating". Younger women reported lower self-efficacy and self-confidence, and being less capable of changing a situation and, consequently, more susceptible to emotional eating. Women with a higher economic level also reported having less self-efficacy. In addition, women with higher BMI reported lower self-efficacy and self-confidence in coping with emotion- and stress-related eating. The goodness-of-fit parameters for the female structural model fitted were $\chi^{2} / d f=4.88 ; \mathrm{CFI}=0.90 ; \mathrm{TLI}=0.90 ; \mathrm{RMSEA}=0.07$, $90 \% \mathrm{CI}=0.067-0.071$. For the male model, higher BMI was significantly associated to lower self-efficacy and self-confidence towards emotional eating. Younger age was associated with less self-efficacy and ability to adapt to challenges. Accordingly, older men had a greater capacity to change a situation, manage emotional reaction, or cope effectively with stressors. The goodness-of-fit parameters for the male structural model fitted were $\chi^{2} / d f=2.82 ; \mathrm{CFI}=0.92$; $\mathrm{TLI}=0.91 ; \mathrm{RMSEA}=0.06,90 \% \mathrm{CI}=0.062-0.069$.

\section{Discussion}

Emotional eating has been associated to obesity [23, 37], eating disorders [10, 37], and other factors, thus investigating such aspect is relevant to reduce these negative outcomes in the population. The use of questionnaires to investigate emotional eating has been shown to be a promising strategy, as it helps to understand affective aspects related to eating. In this way, the EADES can be a tool of interest, as it captures different affective aspects as well as coping strategies related to eating. As this instrument had not yet been used in Brazil, our first aim was to develop a Portuguese version of the EADES. As a second aim, we evaluated the psychometric properties of the EADES when applied in a sample of Brazilian adults to ensure the validity and reliability of the data. Finally, as a third aim, we investigated whether sex, age, economic level, and BMI were associated with the concepts evaluated by the EADES aiming to find groups vulnerable to emotional eating. Thus, this study contributes to the literature by presenting for the first time, to our knowledge, Brazilian data from the EADES, which can be a useful instrument for future clinical and scientific protocols.

The assessment of the validity of the EADES data showed that the original model, which had an adequate fit for an USA sample [7], did not fit the Brazilian sample. On the other hand, after refinement (i.e., deleting items), a factorial model proposed in a Mexican sample [2] showed good psychometric parameters for the Brazilian sample. These results show that a distinct factorial structure of the EADES was fitted for each country, which highlights the importance of investigating the psychometric properties of the instrument before its use [33]. Being this the first study that used the EADES in Brazil, comparisons with Brazilian data were not possible, and future studies are encouraged. However, we suggest that future protocols should be caution in using EADES when its validity and reliability for the application context is unknown. In addition, as countries tend to have different eating cultures, EADES results (i.e., scores of 
Table 2 Standardized factorial loadings $(\lambda)$ of items the Eating and Appraisal due to Emotions and Stress (EADES) Questionnaire of the original and Mexican models to the sample of Brazilian adults (Total sample: $n=1,240$; Female sample: $n=815$; Male sample: $n=425$ )

\begin{tabular}{|c|c|c|c|c|c|c|}
\hline \multirow[b]{2}{*}{ Item } & \multicolumn{6}{|c|}{ Factorial loading $(\lambda)$} \\
\hline & $\begin{array}{l}\text { Original }^{\#} \\
\text { (Total sample) }^{\text {Tal }}\end{array}$ & $\begin{array}{l}\text { Original } \\
\text { (Female sample) }\end{array}$ & $\begin{array}{l}\text { Original } \\
\text { (Male sample) }\end{array}$ & $\begin{array}{l}\text { Mexican }{ }^{\# \#} \\
\text { (Total sample) }\end{array}$ & $\begin{array}{l}\text { Mexican } \\
\text { (Female sample) }\end{array}$ & $\begin{array}{l}\text { Mexican } \\
\text { (Male sample) }\end{array}$ \\
\hline 2 & 0.30 & 0.28 & 0.36 & 0.31 & 0.29 & 0.37 \\
\hline 3 & 0.48 & 0.42 & 0.59 & 0.57 & 0.50 & 0.67 \\
\hline 4 & 0.69 & 0.65 & 0.71 & 0.75 & 0.71 & 0.78 \\
\hline 5 & 0.71 & 0.71 & 0.71 & 0.72 & 0.72 & 0.72 \\
\hline 6 & 0.78 & 0.79 & 0.74 & 0.77 & 0.77 & 0.75 \\
\hline 7 & 0.67 & 0.67 & 0.65 & 0.66 & 0.66 & 0.63 \\
\hline 8 & 0.56 & 0.58 & 0.56 & 0.57 & 0.58 & 0.58 \\
\hline 10 & 0.31 & 0.31 & 0.34 & - & - & - \\
\hline 11 & 0.43 & 0.41 & 0.45 & 0.44 & 0.43 & 0.46 \\
\hline 12 & 0.62 & 0.65 & 0.57 & - & - & - \\
\hline 13 & 0.68 & 0.70 & 0.59 & 0.73 & 0.75 & 0.65 \\
\hline 14 & 0.78 & 0.74 & 0.78 & 0.83 & 0.80 & 0.83 \\
\hline 15 & 0.47 & 0.56 & 0.32 & - & - & - \\
\hline 16 & 0.55 & 0.53 & 0.60 & 0.65 & 0.63 & 0.71 \\
\hline 18 & 0.05 & 0.09 & 0.07 & 0.08 & 0.12 & 0.10 \\
\hline 20 & 0.63 & 0.62 & 0.58 & 0.62 & 0.61 & 0.57 \\
\hline 21 & 0.73 & 0.73 & 0.70 & 0.82 & 0.82 & 0.83 \\
\hline 22 & 0.60 & 0.57 & 0.64 & 0.61 & 0.59 & 0.64 \\
\hline 23 & 0.55 & 0.60 & 0.52 & 0.59 & 0.64 & 0.57 \\
\hline 24 & 0.43 & 0.40 & 0.48 & 0.40 & 0.37 & 0.45 \\
\hline 25 & 0.55 & 0.58 & 0.51 & 0.56 & 0.56 & 0.53 \\
\hline 26 & 0.72 & 0.74 & 0.64 & 0.81 & 0.81 & 0.78 \\
\hline 27 & 0.70 & 0.68 & 0.66 & 0.69 & 0.67 & 0.66 \\
\hline 28 & 0.70 & 0.69 & 0.71 & 0.75 & 0.74 & 0.76 \\
\hline 29 & 0.25 & 0.32 & 0.26 & 0.27 & 0.34 & 0.28 \\
\hline 30 & 0.68 & 0.75 & 0.52 & 0.77 & 0.81 & 0.68 \\
\hline 31 & 0.80 & 0.83 & 0.75 & - & - & - \\
\hline 32 & 0.52 & 0.49 & 0.60 & 0.51 & 0.47 & 0.60 \\
\hline 33 & 0.65 & 0.62 & 0.75 & 0.67 & 0.64 & 0.76 \\
\hline 34 & 0.80 & 0.85 & 0.66 & 0.88 & 0.90 & 0.80 \\
\hline 35 & 0.63 & 0.64 & 0.60 & 0.68 & 0.69 & 0.66 \\
\hline 36 & 0.82 & 0.79 & 0.82 & 0.87 & 0.84 & 0.87 \\
\hline 37 & 0.69 & 0.64 & 0.73 & 0.69 & 0.65 & 0.73 \\
\hline 38 & 0.73 & 0.76 & 0.64 & 0.82 & 0.83 & 0.78 \\
\hline 39 & 0.83 & 0.80 & 0.82 & 0.88 & 0.86 & 0.86 \\
\hline 40 & 0.47 & 0.48 & 0.48 & 0.47 & 0.49 & 0.49 \\
\hline 41 & 0.32 & 0.30 & 0.41 & - & - & - \\
\hline 42 & 0.48 & 0.53 & 0.38 & - & - & - \\
\hline 43 & 0.55 & 0.51 & 0.58 & 0.61 & 0.58 & 0.65 \\
\hline 45 & 0.76 & 0.71 & 0.77 & 0.80 & 0.77 & 0.82 \\
\hline 46 & 0.55 & 0.57 & 0.48 & - & - & - \\
\hline 47 & 0.68 & 0.67 & 0.65 & 0.77 & 0.76 & 0.76 \\
\hline 48 & 0.70 & 0.63 & 0.72 & - & - & - \\
\hline 49 & 0.50 & 0.46 & 0.58 & 0.62 & 0.57 & 0.71 \\
\hline 50 & 0.71 & 0.67 & 0.69 & 0.77 & 0.73 & 0.75 \\
\hline 51 & 0.70 & 0.70 & 0.69 & 0.70 & 0.70 & 0.70 \\
\hline 52 & 0.13 & 0.61 & 0.23 & - & - & - \\
\hline 53 & 0.53 & 0.49 & 0.64 & 0.55 & 0.50 & 0.65 \\
\hline 54 & 0.70 & 0.75 & 0.66 & 0.75 & 0.80 & 0.71 \\
\hline
\end{tabular}


Table 2 (continued)

\#Ozier AD, Kendrick OW, Knol LL, Leeper JD, Perko M, Burnham J. The Eating and Appraisal Due to Emotions and Stress (EADES) Questionnaire: development and validation. J Am Diet Assoc. 2007;107(4):619-28

\#\# Lazarevich I, Irigoyen-Camacho ME, Velazquez-Alva Mdel C, Salinas-Avila J. Psychometric characteristics of the Eating and Appraisal Due to Emotions and Stress Questionnaire and obesity in Mexican university students. Nutricion hospitalaria. 2015;31(6):2437-44

eating due to emotions and stress) are unlikely to be similar across populations. Although we did not test this relationship, it is consistent with the literature [38,39], which shows that culture and society strongly influence people's eating habits and patterns. In this way, the different factorial structures found for EADES could be a reflection of the characteristics of each culture.

Fitting a different model for each sex shows that women and men have different coping strategies involving eating during stressful and emotional situations. In this perspective, our findings corroborate the literature, highlighting important differences in emotional eating between women and men [22-24, 40, 41]. Although the original study did not evaluate separately the EADES factorial model for each sex [2], Kelly et al. [42] suggest that women tend to present a coping strategy focused more on emotion, aiming to change their emotional response to stressors. Men, on the other hand, seem to focus more on the problem itself, aiming to deal or eliminate the stressor [42]. These studies support the different factorial models found in this study.

Our finding on reliability corroborate those presented by Ozier et al. [7] and Lazarevich et al. [2]. Furthermore, the original study found a higher reliability for the first two factors of the instrument ("Emotion- and Stress-Related Eating" and "Appraisal of Ability and Resources to Cope with Emotions and Stress") compared to the third factor ("Appraisal of Outside Stressors/Influences"), which was also observed in the present work. The original authors explain that the lower reliability of the third factor could be due to the smaller number of items (five) and suggest further studies to formulate additional items to increase the factor's reliability, which could also improve the observed low convergent validity. We also found adequate reliability for the total EADES (i.e., all items: $\mathrm{CR} \geq 0.94 ; \alpha>0.92$ ), which corroborates the literature $[2,7]$. These findings demonstrate that the instrument is able to measure, in a consistent way, the proposed construct [33]. Thus, this instrument can help identify individuals who use eating as a way of coping with their emotions and stress and, based on the results, promote behavioral change - as emotional eating is a learned behavior and can be modified - to deal with stressful situations and develop rational coping skills.

Concerning our last objective, the results showed that individual characteristics were associated with the EADES concepts. To our knowledge, no study using the EADES has assessed this association using structural equation modeling, indicating that our findings are unprecedented. First, we found that younger respondents reported higher levels of emotional eating. This finding contrasts with the results presented by the Mexican study [2], in which the ability to cope with emotions and stress decreased with age. On the other hand, Abdella et al. [25] found that younger individuals tend to eat more during hedonic situations when compared to older individuals. Concordantly, Charles and Carstensen [43] found that older individuals seem to have a greater ability to neutralize an emotion when facing stressful situations. These findings highlight the need to help the young and less experienced population to develop greater self-efficacy and self-confidence and prevent the habit of eating to cope with emotional problems [7].

Also, we found a significant association between BMI and self-efficacy and self-confidence in emotions- and stress-related eating in women and men. The higher the BMI the less respondents felt able to control the eating behavior. These data are in accordance with the literature $[2,13,15,23]$ and give support to the inclusion of BMI as a factor in screening protocols to identify individuals susceptible to using food as a coping strategy for emotions and stress. The lower sense of self-efficacy towards emotional eating found in women with higher socio-economic level can be explained by the greater purchasing power of such people giving them more access to a greater variety of foods including the highly processed, fat- and sugar-rich products [27]. We speculate that during stressful episodes, low self-efficacy or lack of control may contribute to higher intake of such products, but we did not find studies that directly compare; therefore, future studies should be carried out to investigate the possible relationship between economic levels and emotional eating. In conclusion, we hope that the individual characteristics found to be significant factors for emotional eating may be useful to support prevention and intervention measures.

\section{What is already known on this subject?}

Emotions and stress can influence people's eating behaviors and cause damage to their physical and mental health. The EADES can be a useful tool to identify these emotions, but little is known about its reliability and validity, especially in Brazilian context. 


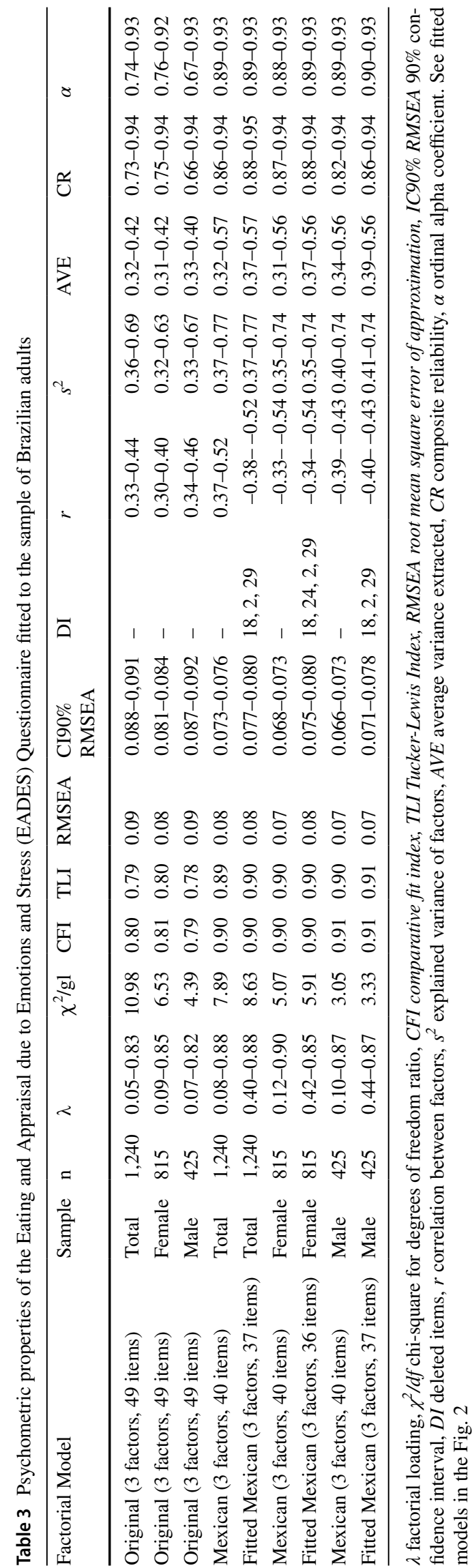

\section{What does this study add?}

A Portuguese language version of the EADES was developed for use in Brazil and different factorial models were fitted for each sex indicating that emotional eating is distinct between women and men. Sex, age, economic level, and BMI were associated to eating and appraisal due to emotions and stress, which can help to direct protocols aimed at improving the people's eating behavior.

\section{Limitations and strengths}

Some limitations of this study should be mentioned. The first was the sampling method based on a non-probabilistic protocol, which limits the representativeness of the results. However, we included a large sample and used robust analytical strategies to partly compensate this limitation. Secondly, the cross-sectional study design, although commonly used and relevant for raising hypotheses, does not allow inference about cause-and-effect relationships. Third, we did not perform an invariance test for different groups to investigate whether the content of the EADES items is understood similarly by people with different characteristics. We, therefore, suggest that future studies include this analysis in their protocols. Despite these limitations, the study also has its strengths. The eating and nutrition process is influenced not only by biological factors, but also by psychological and cultural factors, such as emotions, memories, habits, social status, and several others. However, most nutritional interventions consider only biological factors and disregard all other elements that can influence and modify the diet. Through the present validation of the EADES for the Portuguese language, the concept of emotional eating can be assessed, thus, expanding the view of professionals in the field and contributing to the development of health protocols and diet interventions that are more efficient.

\section{Conclusions}

The Brazilian Portuguese version of the EADES produced was well understood by the young Brazilian adults. The EADES original factorial model did not show good psychometric properties for the Brazilian data. On the other hand, a factorial model proposed for a Mexican context showed good parameters after deleting different items for both female and male samples; therefore, a different factorial model was fitted for each sex and is now available for use in similar populations. Finally, we found that age, economic level, and BMI were associated to eating and appraisal due to emotions and stress and should be considered in future protocols. 
Table 4 Influence of demographic variables on eating related to emotions and stress

\begin{tabular}{|c|c|c|c|c|c|c|c|c|c|c|c|c|c|}
\hline \multirow{2}{*}{\multicolumn{2}{|c|}{ Variable }} & \multicolumn{12}{|c|}{ Structural Model } \\
\hline & & \multicolumn{3}{|c|}{ Initial Female } & \multicolumn{3}{|c|}{ Final Female } & \multicolumn{3}{|c|}{ Initial Male } & \multicolumn{3}{|c|}{ Final Male } \\
\hline Independent & Dependent & $\beta$ & SE & $\mathrm{p}$ & $\beta$ & SE & $\mathrm{p}$ & $\beta$ & $\mathrm{SE}$ & $\mathrm{p}$ & $\beta$ & SE & $\mathrm{p}$ \\
\hline Age & SEESRE & -0.158 & 0.036 & $<0.001 *$ & -0.158 & 0.036 & $<0.001 *$ & -0.219 & 0.051 & $<0.001 *$ & -0.227 & 0.050 & $<0.001 *$ \\
\hline Economic Level & SEESRE & 0.081 & 0.033 & $0.014 *$ & 0.081 & 0.033 & $0.014 *$ & 0.053 & 0.048 & 0.275 & - & - & - \\
\hline BMI & SEESRE & 0.229 & 0.033 & $<0.001 *$ & 0.229 & 0.033 & $<0.001 *$ & 0.262 & 0.047 & $<0.001 *$ & 0.262 & 0.046 & $<0.001 *$ \\
\hline Age & SCESRE & 0.102 & 0.040 & $0.010 *$ & 0.102 & 0.040 & $0.010 *$ & 0.052 & 0.055 & 0.344 & - & - & - \\
\hline Economic Level & SCESRE & -0.004 & 0.034 & 0.912 & - & - & - & 0.068 & 0.049 & 0.168 & - & - & - \\
\hline BMI & SCESRE & -0.093 & 0.035 & $0.009 *$ & -0.093 & 0.035 & $0.008 *$ & -0.131 & 0.052 & $0.011^{*}$ & -0.131 & 0.051 & $0.011 *$ \\
\hline Age & ARAC & 0.239 & 0.037 & $<0.001 *$ & 0.239 & 0.037 & $<0.001 *$ & 0.143 & 0.055 & $0.009 *$ & 0.133 & 0.054 & $0.013 *$ \\
\hline Economic Level & ARAC & -0.003 & 0.035 & 0.933 & - & - & - & 0.070 & 0.051 & 0.169 & - & - & - \\
\hline BMI & ARAC & -0.048 & 0.035 & 0.169 & - & - & - & -0.057 & 0.053 & 0.283 & - & - & - \\
\hline
\end{tabular}

SEESRE Self-efficacy in Emotion- and Stress-Related Eating, SCESRE Self-confidence in Emotion- and Stress-Related Eating, ARAC Appraisal of Resources and Ability to Cope, $\beta$ standardized estimate, $S E$ standard error, ${ }^{*} p<0.05$. The dependent variables represent the factors of the fitted factorial model for the Brazilian sample of the Eating and Appraisal due to Emotions and Stress (EADES) Questionnaire

Supplementary Information The online version contains supplementary material available at https://doi.org/10.1007/s40519-021-01309-y.

Author contributions PCS, WRS, and JADBC participated in the conception and design of the study. PCS wrote the first version of the manuscript. WRS, JM, and JADBC revised the final version of the manuscript. All authors contributed to the analysis and interpretation of data.

Funding This work was supported by the Coordination for the Improvement of Higher Education Personnel-Brazil (CAPESFinancing Code 001) and by the São Paulo Research Foundation (FAPESP, Grant 2019/19590-9).

Data availability Anonymized data and analysis are available from the corresponding author on reasonable request.

Code availability Not applicable.

\section{Declarations}

Conflict of interest The authors have no conflict of interest to declare.

Ethics approval This study was conducted according to the guidelines laid down in the Declaration of Helsinki, and all procedures involving study participants were approved by the Human Research Ethics Committee of the School of Pharmaceutical Sciences of UNESP (C.A.A.E.: 11,469,719.1.0000.5426).

Informed consent All participants signed a consent form.

Consent for publication Not applicable.

\section{References}

1. Macht M (2008) How emotions affect eating: a five-way model. Appetite 50(1):1-11. https://doi.org/10.1016/j.appet.2007.07.002
2. Lazarevich I, Irigoyen-Camacho ME, Velazquez-Alva Mdel C, Salinas-avila J (2015) psychometric characteristics of the eating and appraisal due to emotions and stress questionnaire and obesity in Mexican university students. Nutr Hosp 31(6):24372444. https://doi.org/10.3305/nh.2015.31.6.8960

3. Evers C, Adriaanse M, de Ridder DT, de Witt Huberts JC (2013) Good mood food. Positive emotion as a neglected trigger for food intake. Appetite 68:1-7. https://doi.org/10.1016/j.appet. 2013.04.007

4. APA American Psychology Association. https://dictionary.apa. org/. Accessed Nov 2021

5. Van Strien T, Herman CP, Anschutz DJ, Engels RC, de Weerth C (2012) Moderation of distress-induced eating by emotional eating scores. Appetite 58(1):277-284. https://doi.org/10. 1016/j.appet.2011.10.005

6. Konttinen H, van Strien T, Mannisto S, Jousilahti P, Haukkala A (2019) Depression, emotional eating and long-term weight changes: a population-based prospective study. Int J Behav Nutr Phys Act 16(1):28. https://doi.org/10.1186/s12966-019-0791-8

7. Ozier AD, Kendrick OW, Knol LL, Leeper JD, Perko M, Burnham J (2007) The Eating and Appraisal Due to Emotions and Stress (EADES) Questionnaire: development and validation. J Am Diet Assoc 107(4):619-628. https://doi.org/10.1016/j.jada. 2007.01.004

8. He J, Chen G, Wu S, Niu R, Fan X (2020) Patterns of negative emotional eating among Chinese young adults: a latent class analysis. Appetite 155:104808. https://doi.org/10.1016/j.appet. 2020.104808

9. Sinha R, Jastreboff AM (2013) Stress as a common risk factor for obesity and addiction. Biol Psychiat 73(9):827-835. https://doi. org/10.1016/j.biopsych.2013.01.032

10. Canetti L, Bachar E, Berry EM (2002) Food and emotion. Behav Proc 60(2):157-164. https://doi.org/10.1016/s0376-6357(02) 00082-7

11. Errisuriz VL, Pasch KE, Perry CL (2016) Perceived stress and dietary choices: The moderating role of stress management. Eat Behav 22:211-216. https://doi.org/10.1016/j.eatbeh.2016.06.008

12. Gluck ME, Geliebter A, Hung J, Yahav E (2004) Cortisol, hunger, and desire to binge eat following a cold stress test in obese women with binge eating disorder. Psychosom Med 66(6):876-881. https://doi.org/10.1097/01.psy.0000143637.63508.47 
13. Meule A, Reichenberger J, Blechert J (2018) Development and preliminary validation of the Salzburg Stress Eating Scale. Appetite 120:442-448. https://doi.org/10.1016/j.appet.2017.10.003

14. Arnow B, Kenardy J, Agras WS (1995) The emotional eating scale: the development of a measure to assess coping with negative affect by eating. Int J Eat Disord 18(1):79-90. https://doi.org/ 10.1002/1098-108x(199507)18:1\%3c79::aid-eat2260180109\% 3e3.0.co;2-v

15. Geliebter A, Aversa A (2003) Emotional eating in overweight, normal weight, and underweight individuals. Eat Behav 3(4):341347. https://doi.org/10.1016/s1471-0153(02)00100-9

16. Sultson H, Kukk K, Akkermann K (2017) Positive and negative emotional eating have different associations with overeating and binge eating: Construction and validation of the Positive-Negative Emotional Eating Scale. Appetite 116:423-430. https://doi.org/10. 1016/j.appet.2017.05.035

17. Stunkard AJ, Messick S (1985) The three-factor eating questionnaire to measure dietary restraint, disinhibition and hunger. J Psychosom Res 29(1):71-83. https://doi.org/10.1016/0022-3999(85) 90010-8

18. Hunot C, Fildes A, Croker H, Llewellyn CH, Wardle J, Beeken RJ (2016) Appetitive traits and relationships with BMI in adults: development of the Adult Eating Behaviour Questionnaire. Appetite 105:356-363. https://doi.org/10.1016/j.appet.2016.05.024

19. Martins BG, da Silva WR, Maroco J, Campos J (2021) Psychometric characteristics of the three-factor eating questionnaire-18 and eating behavior in undergraduate students. Eat Weight Disord 26(2):525-536. https://doi.org/10.1007/s40519-020-00885-9

20. Mazzolani BC, Smaira FI, Esteves GP, André HCS, Amarante MC, Castanho D, Campos K, Benatti FB, Pinto AJ, Roschel H, Gualano B, Ferreira CN (2020) Influence of nutritional status on eating habits and food choice determinants among Brazilian women during the COVID-19 pandemic. medrxiv [Preprint]. https://doi.org/10.1101/2020.11.03.20225136

21. Campos JADB, Martins BG, Campos LA, Maroco J, Saadiq RA, Ruano R (2020) Early Psychological Impact of the COVID-19 Pandemic in Brazil: A National Survey. J Clin Med 9(9). https:// doi.org/10.3390/jcm9092976

22. Lauzon B, Romon M, Deschamps V, Lafay L, Borys JM, Karlsson J, Ducimetière P, Charles MA (2004) The three-factor eating questionnaire-R18 is able to distinguish among different eating patterns in a general population. J Nutr 134(9):2372-2380. https:// doi.org/10.1093/jn/134.9.2372

23. Peneau S, Menard E, Mejean C, Bellisle F, Hercberg S (2013) Sex and dieting modify the association between emotional eating and weight status. Am J Clin Nutr 97(6):1307-1313. https://doi.org/ 10.3945/ajen.112.054916

24. Jiang Y, King JM, Prinyawiwatkul W (2014) A review of measurement and relationships between food, eating behavior and emotion. Trends Food Sci Technol 36(1):15-28. https://doi.org/10. 1016/j.tifs.2013.12.005

25. Abdella HM, El Farssi HO, Broom DR (2019) Eating behaviours and food cravings; influence of age, sex, BMI and FTO Genotype. Nutrients 11 (2). https://doi.org/10.3390/nu11020377

26. Pelchat ML (1997) Food cravings in young and elderly adults. Appetite 28(2):103-113. https://doi.org/10.1006/appe.1996.0063

27. Vigitel (2018) Vigilância de fatores de risco e proteção para doenças crônicas por inquérito telefônico. https://portalarquivos2. saude.gov.br/images/pdf/2019/julho/25/vigitel-brasil-2018.pdf. Accessed Nov 2019

28. Hair JF, Black WC, Babin B, Anderson RE, Tatham RL (November, 2009) Multivariate data analysis. vol 7th ed. Prentice Hall, Upper Saddle River

29. de Onis M, Onyango AW, Borghi E, Siyam A, Nishida C, Siekmann J (2007) Development of a WHO growth reference for school-aged children and adolescents. Bull World Health Organ 85(9):660-667. https://doi.org/10.2471/blt.07.043497

30. WHO (2020) Obesity: preventing and managing the global epidemic. World Health Organization: WHO: Technical Report Series, Geneva

31. ABEP (2020) Brazilian Economic Classifcation Criteria (Brazilian Criteria). https://www.abep.org/criterio-brasil. Accessed Nov 2020

32. Beaton D, Bombardier C, Guillemin F, Ferraz MB (2002) Recommendations for the Cross-Cultural Adaptation of Health Status Measures American Academy of Orthopaedic Surgeons and Institute for Work \& Health. 36

33. Marôco J (2014) Análise de equações estruturais, 2nd ${ }^{\mathrm{a}}$ edn. Report Number, Pêro Pinheiro

34. Fornell C, Larcker DF (1981) Evaluating structural equation models with unobservable variables and measurement error. J Mark Res 18:39-50. https://doi.org/10.2307/3151312?uid=3737664\& uid $=2 \&$ uid $=4 \&$ sid $=21103223270061$

35. Rossel Y (2012) An R package for structural equation modeling. Version 0.5-12 (BETA). J Stat Software 48(2):1-36. https://doi. org/10.18637/jss.v048.i02

36. Jorgensen TD, Pornprasertmanit S, Schoemann AM, Rosseel Y (2018) semTools: Useful tools for structural equation modeling. https://CRAN.R-project.org/package $=$ semTools

37. Singh M (2014) Mood, food, and obesity. Front Psychol 5:925. https://doi.org/10.3389/fpsyg.2014.00925

38. Naska A, Fouskakis D, Oikonomou E, Almeida MD, Berg MA, Gedrich K, Moreiras O, Nelson M, Trygg K, Turrini A, Remaut AM, Volatier JL, Trichopoulou A, DAFNE participants, (2006) Dietary patterns and their socio-demographic determinants in 10 European countries: data from the DAFNE databank. Eur J Clin Nutr 60(2):181-190. https://doi.org/10.1038/sj.ejcn.1602284

39. Gómez G, Fisberg RM, Nogueira Previdelli Á, Hermes Sales C, Kovalskys I, Fisberg M, Herrera-Cuenca M, Cortés Sanabria LY, García MCY, Pareja Torres RG, Rigotti A, Guajardo V, Zalcman Zimberg I, Chinnock A, Murillo AG, Brenes JC, Elans Study Group OBOT (2019) Diet quality and diet diversity in eight Latin American countries: results from the Latin American Study of Nutrition and Health (ELANS). Nutrients. 11(7):1605. https:// doi.org/10.3390/nu11071605

40. Camilleri GM, Méjean C, Kesse-Guyot E, Andreeva VA, Bellisle F, Hercberg S, Péneau S (2014) The associations between emotional eating and consumption of energy-dense snack foods are modified by sex and depressive symptomatology. J Nutr 144(8):1264-1273. https://doi.org/10.3945/jn.114.193177

41. Silva WR, Swami V, Neves AN, Marôco J, Ochner CN, Campos JADB (2019) The Body shape questionnaire is not invariant across sex: evidence from Portuguese-Speaking University students. Percept Mot Skills 126:462-476. https://doi.org/10.1177/0031512519 839537

42. Kelly MM, Tyrka AR, Price LH, Carpenter LL (2008) Sex differences in the use of coping strategies: predictors of anxiety and depressive symptoms. Depress Anxiety 25(10):839-846. https:// doi.org/10.1002/da.20341

43. Charles ST, Carstensen LL (2010) Social and emotional aging. Annu Rev Psychol 61:383-409. https://doi.org/10.1146/annurev. psych.093008.100448

Publisher's Note Springer Nature remains neutral with regard to jurisdictional claims in published maps and institutional affiliations. 